\title{
GAIA and the Extragalactic Distance Scale
}

\author{
G.A. Tammann and B. Reindl \\ Astronomisches Institut der Universität Basel \\ Venusstrasse 7, CH-4102 Binningen, Switzerland
}

\begin{abstract}
The local expansion field $\left(v_{220}<1200 \mathrm{~km} \mathrm{~s}^{-1}\right)$ and the cosmic expansion field out to $30000 \mathrm{~km} \mathrm{~s}^{-1}$ are characterized by $H_{0}=58\left[\mathrm{~km} \mathrm{~s}^{-1} \mathrm{Mpc}^{-1}\right]$. While the random error of this determination is small ( \pm 2 units), it may still be affected by systematic errors as large as $\pm 10 \%$. The local expansion is outlined by Cepheids and by Cepheid-calibrated TF distances of a complete sample of field galaxies and by nearby groups and clusters; the cosmic expansion is defined by Cepheid-calibrated SNeIa. The main source of systematic errors are therefore the shape and the zero point of the P-L relation of Cepheids and its possible dependence on metallicity. GAIA will essentially eliminate these systematic error sources. Another source of systematic error is due to the homogenization of SNeIa as to decline rate $\Delta m_{15}$ and color $(B-V)$. GAIA will discover about half of the $2200 \mathrm{SNe}$ Ia which will occur during a four-year lifetime within $10000 \mathrm{~km} \mathrm{~s}^{-1}$. For many of them groundbased follow-up will provide useful photometric parameters (and spectra), which will allow to fix the dependence of the SNe Ia luminosity on $\Delta m_{15}$ and $(B-V)$ with high accuracy. At the same time they will yield exquisite distances to a corresponding number of field galaxies. - GAIA will also revolutionize the very local distance scale by determining fundamental distances of the companion galaxies of the Milky Way and even of some spirals in- and possibly outside the Local Group from their rotation curves seen in radial velocities and proper motions.

Moreover, GAIA will obtain trigonometric parallaxes of RR Lyrae stars, of red giants defining the TRGB, of stars on the ZAMS, of White Dwarf defining their cooling sequence, and of globular clusters, and determine the metallicity dependence of these distance indicators. It will thus establish a self-controlling network of distance indicators within the Local Group and beyond.
\end{abstract}

\section{Introduction}

An evaluation of GAIA's contribution to the extragalactic distance scale requires as a first step a brief description of the present situation (Section 2). Inadequacies of the present situation of the distance scale are discussed in Section 3. Important improvements expected from GAIA are outlined in Section 4. Some conclusions are given in Section 5.

\section{Status Quo}

The two pillars of the extragalactic distance scale are Cepheids and supernovae of type Ia, which are discussed in turn, as well as other distance indicators.

(c) 2018 Kluwer Academic Publishers. Printed in the Netherlands. 


\subsection{Cepheids}

The fundamental rôle of classical Cepheids for extragalactic distances rests on their period-luminosity (P-L) relation, whose shape and zero point must be known.

\subsubsection{The shape of the P-L relation}

Most published Cepheid distances are based on a linear, wavelengthdependent P-L relation as derived by Madore \& Freedman (1991) from LMC Cepheids:

$$
\begin{aligned}
M_{B} & =-2.43 \log P-1.13 \\
M_{V} & =-2.76 \log P-1.46 \\
M_{I} & =-3.06 \log P-1.87
\end{aligned}
$$

(As to the zero point see 2.1.2.).

These equations yield apparent moduli $\mu_{B}, \mu_{V}$, and $\mu_{I}$, still affected by Galactic, and in the case of extragalactic Cepheids by internal absorption. The true distance modulus $\mu^{0}$ can be obtained by a combination of the apparent moduli if an absorption law is adopted (here: $A_{B}=4.1 E_{B-V}, A_{V}=3.1 E_{B-V}$, and $\left.A_{I}=1.8 E_{B-V}\right)$, i.e.

$$
\begin{aligned}
& \mu^{0}=4.1 \mu_{V}-3.1 \mu_{B} \\
& \mu^{0}=2.38 \mu_{I}-1.38 \mu_{V} .
\end{aligned}
$$

Almost all galaxies with Cepheid distances outside the Local Group have been observed with HST in $V$ and $I$. If one assumes (optimistically) that the apparent moduli $\mu_{V}$ and $\mu_{I}$ have errors of 0.05 the typical error of a Cepheid modulus $\mu_{0}$ becomes 0.15 (7\% in linear distance) from equation (5). - If the Cepheids in a galaxy suffer variable absorption they can be reduced one by one with equations (4) and (5); their true distances can then be averaged.

The OGLE programme has provided new $B, V$, and $I$ photometry for hundreds of fundamental-mode LMC Cepheids (Udalski et al. 1999) which requires a significant change of slope of the P-L relations at periods of 10 days (cf. Fig. 1)

$$
\begin{aligned}
P<10 \text { days }: M_{B} & =(-2.42 \pm 0.08) \log P-(1.24 \mp 0.05) ; \\
M_{V} & =(-2.86 \pm 0.05) \log P-(1.46 \mp 0.03) ; \\
M_{I} & =(-3.03 \pm 0.03) \log P-(1.96 \mp 0.02) \\
P>10 \text { days }: M_{B} & =(-1.89 \pm 0.62) \log P-(1.71 \mp 0.74) ; \\
M_{V} & =(-2.48 \pm 0.17) \log P-(1.81 \mp 0.20) ; \\
M_{I} & =(-2.82 \pm 0.13) \log P-(2.15 \mp 0.154) .
\end{aligned}
$$




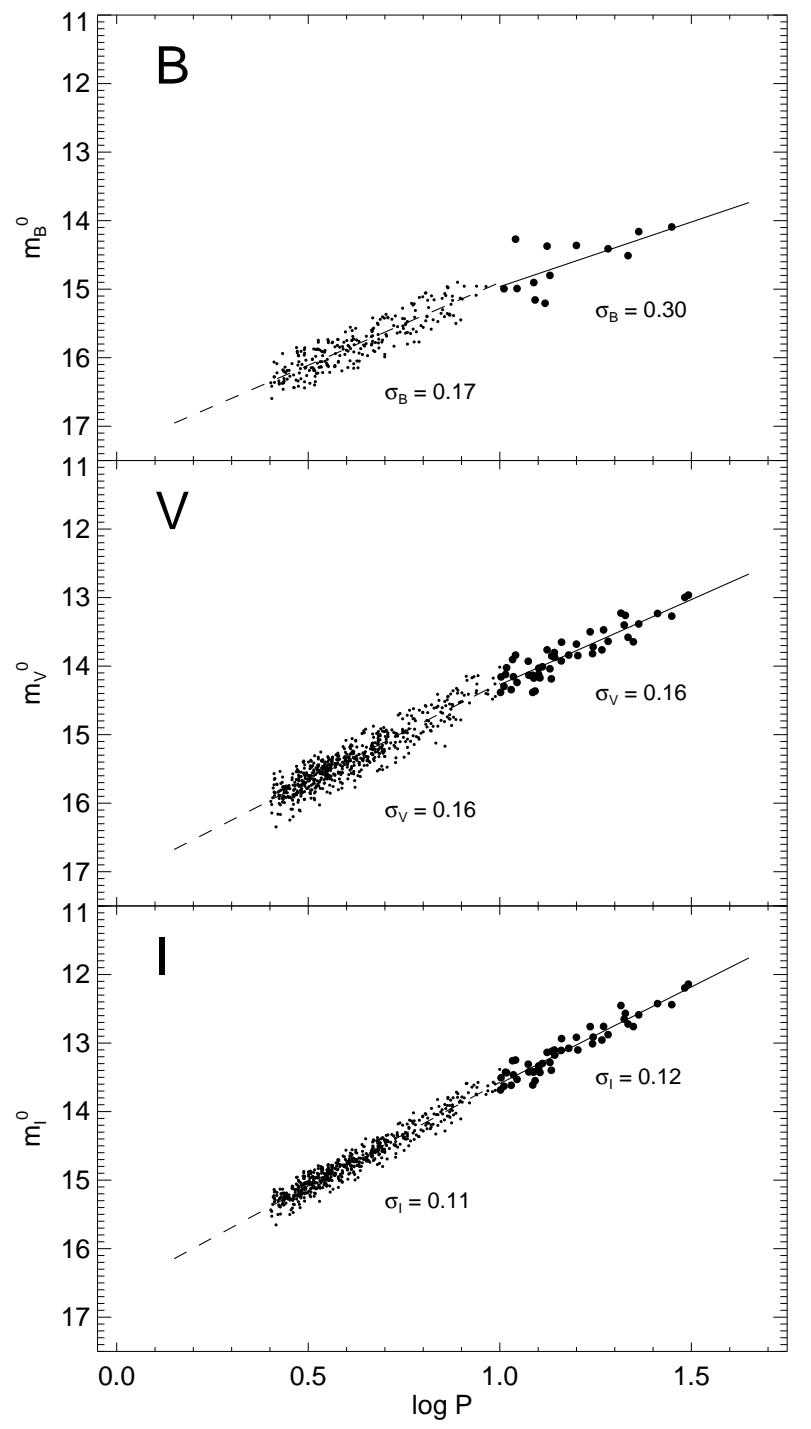

Figure 1. $P$ - $L$ relations in $B, V$, and $I$ of the fundamental-mode LMC Cepheids adopted by Udalski et al. (1999). Small symbols: $P<10^{\mathrm{d}}$; large symbols: $P>10^{\mathrm{d}}$.

A corresponding change of slope is also seen in the period-color $(\mathrm{P}-\mathrm{C})$ relations (cf. Fig. 2).

Most of the available extra-Local Group Cepheid distances rely on variables with $P>10^{\mathrm{d}}$. Their distances derived from equation (10) and (11) are smaller by

$$
\Delta \mu=-0.18 \log P+0.14,
$$




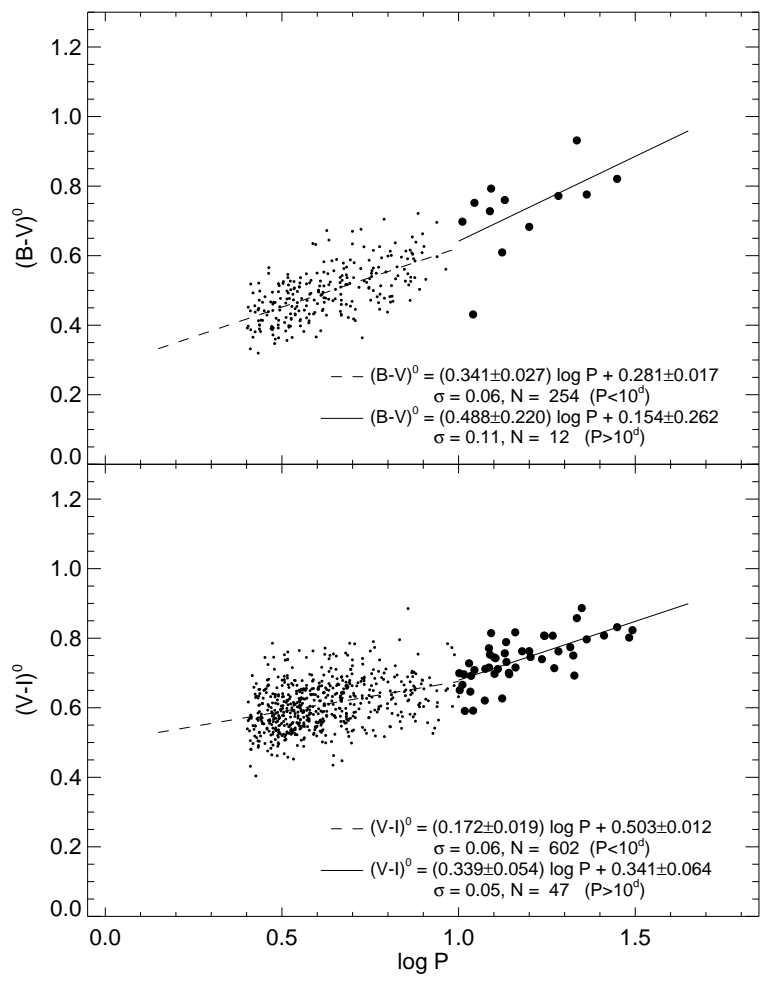

Figure 2. The $(B-V)$ and $(V-I)$ vs. $\log P$ diagrams of the fundamental-mode LMC Cepheids adopted by Udalski et al. (1999). Color excesses from that source. Symbols like in Fig. 1.

as those from equations (2) and (3). All following distances are here still based on the conventional P-L relations in equations (2) and (3).

\subsubsection{The zero point of the $P$ - $L$ relation}

The zero point of the P-L relations in equations (1) - (3) (as well as the one in equations $6-11$ ) is based on an adopted LMC distance modulus of $(m-M)_{\mathrm{LMC}}=18.56$ (cf. Table I). This value is the straight mean of various methods to determine the LMC distance. A straight mean is preferred here because the systematic errors are quite uncertain.

\subsubsection{Cepheids and the local expansion field}

Within the Local Group Cepheid distances have been determined for M 31, M 33, NGC 6822, IC 1613 and a few additional irregular galaxies. Beyond the Local Group one has Cepheid distances for 31 galaxies as compiled in Tammann et al. (2001), the bulk of those being observed with HST. The subset of 23 galaxies outside the noisy Virgo region, i.e. 
Table I. The Distance Modulus of LMC

\begin{tabular}{|c|c|c|}
\hline Reference & $\mu^{0}$ & Method \\
\hline Sandage \& Tammann (1971) & 18.59 & Galactic Cepheids in Clusters \\
\hline Feast (1984) & 18.50 & Cepheids, OB, RR Lyrae, MS-fitting, Mira \\
\hline Feast \& Catchpole (1997) & $18.70 \pm 0.10$ & HIPPARCOS parallaxes of Galactic Cepheids \\
\hline Van Leeuwen et al. (1997) & $18.54 \pm 0.10$ & HIPPARCOS parallaxes of Miras \\
\hline Madore \& Freedman (1998) & $18.44-18.57$ & HIPPARCOS parallaxes of Galactic Cepheids \\
\hline Panagia (1999) & $18.58 \pm 0.05$ & Ring around SN 1987A \\
\hline Pont (1999) & $18.58 \pm 0.05$ & HIPPARCOS parallaxes of Galactic Cepheids \\
\hline Walker (1999) & $18.55 \pm 0.10$ & Review \\
\hline Feast (1999) & $18.60 \pm 0.10$ & Review \\
\hline Walker (1992), Udalski et al. (1999) & $18.53 \pm 0.08$ & $\begin{array}{l}M_{V}^{\mathrm{RR}}=0.41 \pm 0.07 \text { Sandage (1993), } \\
\text { Chaboyer et al. }(1998)\end{array}$ \\
\hline Groenewegen \& Oudmaijer (2000) & $18.60 \pm 0.11$ & HIPPARCOS parallaxes of Galactic Cepheids \\
\hline Kovács (2000) & 18.52 & Double mode RR Lyr \\
\hline Sakai et al. (2000) & $18.59 \pm 0.09$ & Tip of RGB \\
\hline Cioni et al. (2000) & $18.55 \pm 0.04$ & Tip of RGB \\
\hline Romaniello et al. (2000) & $18.59 \pm 0.09$ & Tip of RGB \\
\hline Groenewegen \& Salaris (2001) & $18.42 \pm 0.07$ & Eclipsing binary \\
\hline Girardi \& Salaris (2001) & $18.55 \pm 0.05$ & Red clump stars \\
\hline Baraffe \& Alibert (2001) & $18.60-18.70$ & Pulsation theory of Cepheids \\
\hline Adopted & $18.56 \pm 0.02$ & \\
\hline
\end{tabular}

$\alpha_{\mathrm{M} 87}>30^{\circ}$, define a Hubble diagram out to $v_{220} \approx 1500 \mathrm{~km} \mathrm{~s}^{-1 \dagger}$ with a scatter of $\sigma_{\mu}=0.43$ (Fig. 3 below), part of which is due to errors of the Cepheid distances, the other part must be attributed to peculiar motions.

\subsection{Other Distance Indicators}

\subsubsection{RR Lyrae stars}

The RR Lyrae stars are important to check the distance of LMC and SMC and other late- and early-type companions of the Milky Way. The calibration of their luminosity is difficult because it depends on metallicity, i.e.

$$
M_{V}=a+b[\mathrm{Fe} / \mathrm{H}] .
$$

A thorough discussion of the calibration through main-sequence fitting of globular clusters, the Cepheid distance of LMC, and the Baade-

\footnotetext{
$\dagger$ The $v_{220}$ velocities are corrected for a self-consistent Virgocentric infall model with a local infall vector of $220 \mathrm{~km} \mathrm{~s}^{-1}$ (Kraan-Korteweg 1986).
} 
Wesselink-Becker method has led Chaboyer et al. (1998) to adopt $a=$ $0.39( \pm 0.1)$ at $[\mathrm{Fe} / \mathrm{H}]=-1.9$ and $b=0.23 \pm 0.04$.

\subsubsection{The Tip of the Red Giant Branch (TRGB)}

The TRGB in the $I$-band has proved to be a useful (nearly) metalindependent distance indicator out to $\sim 10 \mathrm{Mpc}$ and eventually to 20 Mpc. For a compilation of 11 available TRGB distances see Kennicutt et al. (1998). The position of the TRGB at $M_{I}=-4.0 \pm 0.1$ depends on the adopted distances of globular cluster (Da Costa \& Armandroff 1990). The method is promising because it can be applied to early- and late-type galaxies and the observational effort is smaller than for Cepheids, but giving about equal distance errors of $\sim 0{ }^{\mathrm{m}} 1-0 .{ }^{\mathrm{m}} 2$.

Also Red Giant Clump stars have been proposed as distance indicators, but their position is metal- and age-dependent, and their application is complex (Girardi \& Salaris 2001).

\subsubsection{Tully-Fisher distances of field galaxies}

The Tully-Fisher (TF) relation, i.e. the correlation of the $21 \mathrm{~cm}$ (or any optical) line width $w$ (taken at $20 \%$ maximum intensity as a measure of the maximum rotation velocity) of a spiral galaxy with its luminosity, has found a wide application. Its latest form is given by (Tammann et al. 2001)

$$
M_{\mathrm{B}}^{0}=-7.31 \log w_{20}-(1.833 \pm 0.095) ; \quad \sigma_{\mathrm{M}}=0.53,
$$

where the slope is taken from a complete volume-limited sample of (favorably inclined) Virgo cluster spirals, while the zero point depends entirely on 29 Cepheid distances; most of the galaxies were observed during an HST Key Project (cf. Freedman et al. 2001).

Equation (14) can be applied to a complete sample of 154 spirals with $v_{220} \leq 1000 \mathrm{~km} \mathrm{~s}^{-1}$ and inclination $i>45^{\circ}$ (Federspiel 1999). The resulting TF distance moduli of the 92 galaxies outside the Virgo region

$\left(\alpha_{\mathrm{M} 87}>30^{\circ}\right)$ are shown in the distance-calibrated Hubble diagram of Fig. 3. Since the dense Virgo region $\left(\alpha_{\mathrm{M} 87}<30^{\circ}\right)$ proper has large peculiar motions, the dynamically defined distance limit becomes fuzzy here causing large scatter and systematic selection effects.

Even outside the Virgo region the scatter about the Hubble line is as large as $\sigma_{(m-M)}=0.86$. Peculiar motions introduce a scatter of $\sigma_{(m-M)}<0.43$ as judged from Cepheids (Section 2.1.3), and additional scatter is introduced by observational errors of the line widths $w$, which were compiled from various sources, and the inclination-dependent absorption corrections. But it is clear, that the intrinsic scatter of the $\mathrm{TF}$ distances cannot be smaller than $\sigma_{(m-M)} \sim 0.5$ (cf. equation 14). This large scatter introduces severe selection bias (Malmquist effect), 
- a problem well known to workers with trigonometric parallaxes. If the above sample of 92 galaxies is cut by an additional apparentmagnitude limit, the scatter decreases and the value of $H_{0}$ increases; the brighter the magnitude cut the more severe the effect becomes (cf. Tammann et al. 2001, Fig. 6). This explains why claims in the literature for an unrealistically small scatter of the TF relation - as derived from incomplete samples - are always accompanied by too large values of $H_{0}$.

The $1000 \mathrm{~km} \mathrm{~s}^{-1}$ sample of TF distances is so far the deepest complete, distance-limited sample of field galaxies for which TF data are available. Sophisticated methods have therefore been developed to correct magnitude-limited samples for Malmquist bias (Theureau et al. 1998; Federspiel et al. 1998; Hendry 2001). But the results become less convincing as the samples thin out with increasing distance.

It has also been proposed to determine distances of field galaxies through the $\mathrm{D}_{\mathrm{n}}-\sigma$ (or fundamental-plane) method, surface brightness fluctuations $(\mathrm{SBF})$ and brightest planetary nebulae. The latter method is confined to early-type galaxies because of the confusing effect of HII regions in late-type galaxies. The same restriction holds for the two first-mentioned methods, unless one assumes that the bulges of spiral galaxies follow the same relations as early-type galaxies. This raises the problem of calibration: there is no normal E/S0 galaxy within $10 \mathrm{Mpc}$ and correspondingly none with a primary distance determination. A meaningful calibration must therefore be based on an adopted Virgo cluster distance and on a fair and sufficiently large sample of cluster members to beat the considerable depth effect of the cluster. An equally severe problem is that no complete magnitude-limited, let alone distance-limited sample of $\mathrm{D}_{\mathrm{n}}-\sigma, \mathrm{SBF}$, or planetary nebulae distances is available at present. The true intrinsic scatter of these three methods is therefore unknown, and the distances so far available cannot be assigned a confidence interval.

An interesting second distance ladder may emerge, based solely on Population II objects. This depends on the supposed universality of the peak of the bell-shaped luminosity function of globular clusters (GCLF). The method has its successes and failures (Tammann \& Sandage 1999 and references therein). The failures may be due to the difficulty to observe the faint descending branch of the GCLF, but on the other hand the method has no physical basis and is purely heuristic. The calibration of the peak luminosity, i.e. $M_{B}=-6.93 \pm 0.08$, rests at present on the RR Lyrae distances of Galactic GC and on the Cepheid distance of M 31. The latter can be substituted by the TRGB distance, which is almost identical (Kennicutt et al. 1998), but calibrated by old objects. 


\subsection{Supernovae of Type IA}

A complete sample of 26 SNe Ia can be established with good CCD photometry in $B, V$ (and $I$ ) near maximum. They fulfill the additional conditions of having $1200<v_{220} \lesssim 30000 \mathrm{~km} \mathrm{~s}^{-1}$, to be blue at maximum, i.e. $(B-V) \leq 0{ }^{\mathrm{m}} 06$, to have Branch-normal spectra as far as known (Branch, Fisher \& Nugent 1993), and to be corrected for Galactic absorption by less than 0.2 (to minimize errors in the absorption correction). If the maximum magnitudes in $B, V$, and $I$ of these SNe Ia are homogenized as for decline rate $\Delta m_{15}$ and color $(B-V)$ (for details see Parodi et al. 2000), they define Hubble diagrams of the form

$$
\log v=0.2 m_{B, V, I}^{\mathrm{corr}}+c_{B, V, I}
$$

with

$$
c_{B}=0.662 \pm 0.005, \quad c_{V}=0.661 \pm 0.005, \quad c_{I}=0.604 \pm 0.005
$$

The scatter amounts in all three relations to only $\sigma_{\mathrm{m}}=0 . \mathrm{m} 11$ ! This small "Hubble" scatter is unparalleled. It can be fully accounted for by observational errors and by a (smaller) contribution from peculiar motions. The intrinsic luminosity scatter of SNe Ia, once they are corrected for decline rate and color, is therefore below the present detection limit. SNe Ia are therefore the best standard candles known. A single SNe Ia yields the distance of any galaxy to within \pm 0.11 (5\%), i.e. even better than a sample of Cepheids. The small luminosity scatter of SNe Ia makes them at the same time practically immune to selection bias.

Consequently the problem of the large-scale distance scale can be solved by equation (15) if only the absolute magnitude of SNeIa is known. For this purpose a specific HST programme has been mounted to determine Cepheid distances of the nearby galaxies which have produced a SNe Ia. To date nine Cepheid-calibrated SNe Ia are known (Saha et al. 2001). Their mean absolute magnitudes are

$<M_{B}^{\text {corr }}>=-19.56 \pm .07,\left\langle M_{V}^{\text {corr }}>=-19.53 \pm .06, \quad<M_{I}^{\text {corr }}>=-19.25 \pm .09\right.$.

The large-scale value of $H_{0}$ (out to distances of $30000 \mathrm{~km} \mathrm{~s}^{-1}$ ) follows by transforming equation (15) to

$$
\log H_{0}=0.2 M_{B, V, I}+c_{B, V, I}+5
$$

and by inserting $M$ and $c_{\lambda}$ from equations (17) and (16), respectively, to yield a mean value of $H_{0}(B, V, I)=56.6 \pm 2.3$. The linear fit to the $\mathrm{SNe}$ Ia data corresponds closely to a $\Omega_{\text {Matter }}=1$ model. If a $\Omega_{\text {Matter }}=$ 
Table II. Distances of local groups and clusters

\begin{tabular}{|c|c|c|c|}
\hline Group/Cluster & $<v_{220}>$ & $\mu^{0}$ & Method \\
\hline South Polar gr.* & 112 & $26.68 \pm 0.20$ & Cepheids $(1)^{\mathrm{a}}$ \\
\hline M 101 gr.* $^{*}$ & 405 & $29.36 \pm 0.10$ & Cepheids $(1)^{\mathrm{a}}$, TRGB $^{\mathrm{f}}$ \\
\hline Leo* & 652 & $30.21 \pm 0.05$ & SNe Ia $(2)^{\mathrm{b}}$, Cepheids $(3)^{\mathrm{a}}$, TRGB $^{\mathrm{f}}$ \\
\hline $\mathrm{UMa}^{\dagger}$ & 1060 & $\geq 31.33 \pm 0.15$ & $\mathrm{TF}^{\mathrm{c}}, \mathrm{LC}^{\mathrm{d}}$ \\
\hline Virgo $\ddagger$ & 1179 & $31.60 \pm 0.20$ & SNe Ia $(3)^{\mathrm{b}}$, Cepheids $(4)^{\mathrm{a}}, \mathrm{TF}^{\mathrm{c}}, \mathrm{LC}^{\mathrm{d}}, \mathrm{GC}^{\mathrm{e}}$ \\
\hline Fornax* & 1338 & $31.60 \pm 0.10$ & SNe Ia $(3)^{\mathrm{b}}$, Cepheids $(3)^{\mathrm{a}}$ \\
\hline Coma & 7188 & $35.31 \pm 0.22$ & $3^{\mathrm{m}} 71 \pm 0.08$ more distant than Virgo ${ }^{\mathrm{g}}$ \\
\hline
\end{tabular}

* Group/cluster membership as defined by Kraan-Korteweg (1986)

$\dagger$ Two separate agglomerations can be distinguished in the UMa cluster; the nearer, presumably more nearly complete agglomeration with $18 \mathrm{TF}$ distances is considered here (Federspiel 1999)

$\ddagger$ Cluster membership as defined by Binggeli et al. (1993)

Sources: (a) For a list of individual Cepheid distances see Tammann et al. (2001) (the number of Cepheid distances is shown in parentheses); (b) SNe Ia calibration as in Section 2.3 (the number of SNe Ia is shown in parentheses); (c) Federspiel (1999); (d) Luminosity classes (Sandage 2001); (e) Globular Clusters (Tammann \& Sandage 1999);

(f) Kennicutt et al. (1998); (g) Tammann \& Sandage (1999)

0.3, $\Omega_{\Lambda}=0.7$ model is preferred $H_{0}$ is slightly increased to $57.4 \pm 2.3$ (cf. Carroll et al. 1992). The individual distances of the sample of $26+$ $9^{\dagger} \mathrm{SNe}$ Ia are determined from their observed maximum magnitudes $m_{B, V, I}$ and from equation (17). They are plotted in Fig. 3 (below).

\subsection{Distances of Local Groups and Clusters}

It can be expected that local galaxy groups and clusters delineate the Hubble flow particularly well because (1) their distances can be based on several distance determinations, and (2) the mean velocity of the cluster members minimizes the peculiar motions. A self-explanatory list of seven local (and not so local) group/cluster distances is given in Table II. Leo has two, Fornax three excellent SNe Ia distances; the Virgo distance from three SNe Ia is not quite as secure because of the considerable depth of the cluster. Six entries in Table II include distances based on one or more galaxies with Cepheids. The TF distance of Virgo is based on a complete sample of 49 suitable spirals; the TF sample of UMa may not be complete and the resulting distance is rather a lower limit.

The seven groups/clusters within $v_{220}<1200 \mathrm{kms}^{-1}$ in Table II define a Hubble diagram with $H_{0}=57.5 \pm 2.1$ (giving half weight to

${ }^{\dagger}$ The 9 SNeIa have a Galactic absorption correction of $A_{V}>0.2$ and are not used for the calibration in equation (16). 


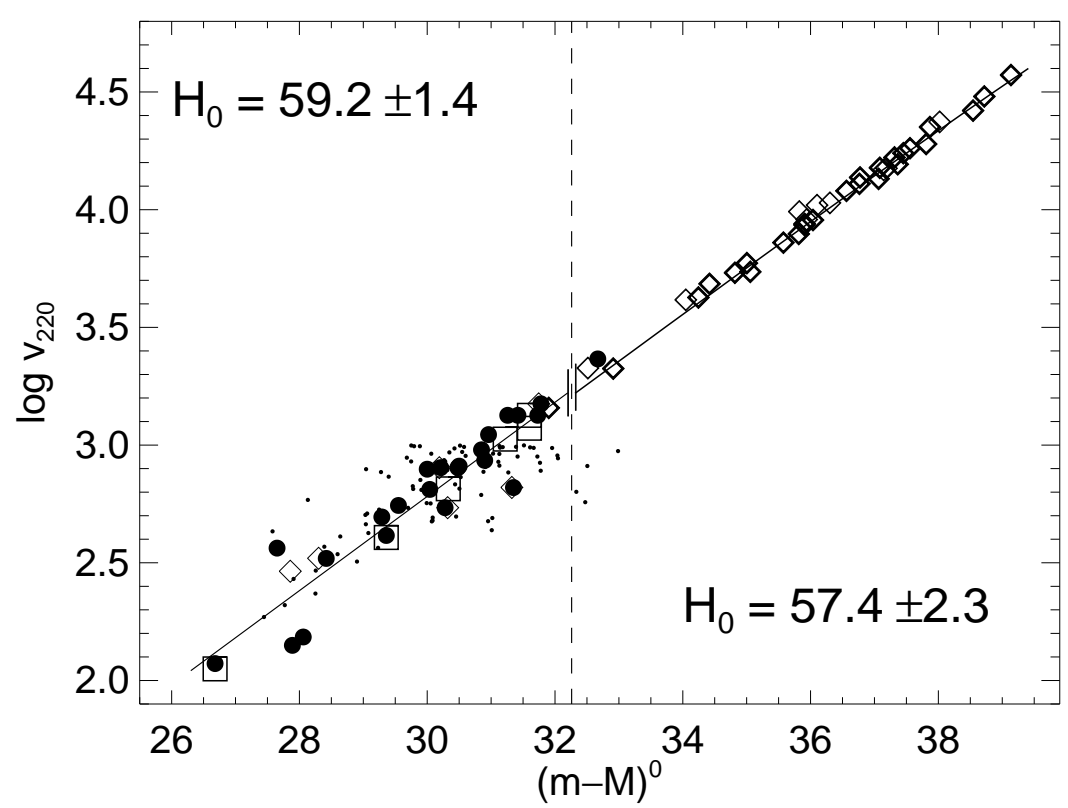

Figure 3. A synoptic distance-calibrated Hubble diagram extending over 11 magnitudes or a factor of 100 in recession velocity. The left-hand value of $H_{0}$ based on Cepheids (large dots), TF distances (small dots), and groups/clusters (squares) is independent of the right-hand value of $H_{0}$ based on SNeIa (diamonds), except that all distances rest ultimately on Cepheids. The full drawn line corresponds to $\Omega_{\text {Matter }}=0.3, \Omega_{\Lambda}=0.7$.

the nearby South Polar group) with a scatter of only $\sigma_{(m-M)}=0.17$. If also the Coma cluster is included one obtains $H_{0}=58.2 \pm 1.8, \sigma_{(m-M)}=$ 0.16 .

\subsection{Summary}

The distances discussed in sections 2.1 to 2.4 are plotted in a distance calibrated Hubble diagram (Fig. 3). They are best fit by a local value of $H_{0}\left(<1200 \mathrm{~km} \mathrm{~s}^{-1}\right)=59.2 \pm 1.4$ and a large-scale value out to $\sim$ $30000 \mathrm{~km} \mathrm{~s}^{-1}$ of $H_{0}($ cosmic $)=57.4 \pm 2.3$. The difference between the local and cosmic value of $H_{0}$ is insignificant. This demonstrates that the expansion rate changes very little with scale. 


\section{Desiderata}

One could gain the impression from Section 2 that the problem of extragalactic distances was solved. Nothing is further from the truth. Only about 200 galaxies with random individual distance errors between 0.1 and 0.5 were involved. For the vast majority of galaxies one has still no distances. Of course, a dynamical distance can be obtained from the recession velocity and an adopted value of $H_{0}$, but the accuracy is limited by peculiar motions. If one assumes $630 \mathrm{~km} \mathrm{~s}^{-1}$ as a typical large-scale peculiar motion, as judged by the local motion with respect to the microwave background, the dynamical distances of galaxies beyond $10000 \mathrm{~km} \mathrm{~s}^{-1}$ are affected by only $\lesssim 6 \%$ from peculiar motions. For galaxies at $3000 \mathrm{~km} \mathrm{~s}^{-1}$ the dynamical distance error is roughly tripled, but stays at this value for still nearer galaxies because the peculiar motions tend to decrease over shorter scales. The "Hubble" scatter of the Cepheid distances suggests that $v_{\text {pec }} / v_{220} \sim 0.2$ (cf. Section 2.1.3). Of course, the dynamical distances of very nearby galaxies $\left(v_{220} \lesssim 200 \mathrm{~km} \mathrm{~s}^{-1}\right.$ or $\left.\lesssim 4 \mathrm{Mpc}\right)$ are highly unreliable, and they require "honest" distance determinations.

This picture is over-optimistic for galaxies in high-density regions (like the Virgo region) because the peculiar motions are larger here.

Some investigations depend just on a comparison of dynamical distances and "honest" distance determinations, e.g. if the infall into the Virgo cluster should be studied, or generally if the local and not so local expansion field should be mapped, which would result, among other things, in a delineation of the co-moving volume which partakes in the local motion towards the microwave background.

Just for this purpose the data in Fig. 3 are insufficient because there are only four SNeIa in the most relevant range $1200<v_{220}<$ $10000 \mathrm{~km} \mathrm{~s}^{-1}$ and not a single SNeIa in the range $2500<v_{220}<$ $4000 \mathrm{~km} \mathrm{~s}^{-1}$ ! For the velocity mapping relative $\mathrm{TF}$ and $\mathrm{D}_{\mathrm{n}}-\sigma$ distances of clusters can close the gap. Giovanelli et al. (1997) and Dale et al. (1999) have derived TF distances of 71 clusters out to $v<$ $25000 \mathrm{~km} \mathrm{~s}^{-1}$. They define a tight Hubble line with a scatter $\left(\sigma_{(m-M)}=\right.$ 0.11) rivalling SNe Ia. But they do not yield an independent value of $\mathrm{H}_{0}$; this is because the individual cluster distances are the mean of about ten subjectively selected cluster members. They are far from constituting a complete sample, which is the conditio sine qua non for any application of equation (14). Also the relative $D_{n}-\sigma$ distances of ten clusters by Kelson et al. (2000) cannot independently be put on an absolute scale for the same reason (cf. Tammann 2001).

In the preceding pages only random statistical errors were quoted, although systematic errors are probably prevailing. Most serious are of 
course systematic errors of the P-L relation of Cepheids because the local expansion field on the left side of Fig. 3 as well as the largescale expansion field on the right side depend on the goodness of the calibrating Cepheid distances.

\subsection{Systematic errors of the Cepheid distances}

\subsubsection{The shape of the $P$ - $L$ relation}

So far all Cepheid distances are based on equations (2) and (3). If equations (10) and (11) were used instead they would be reduced by 0.13 or $6 \%$ (equation 12) on average, assuming a median period of $30^{\mathrm{d}}$ for the Cepheids involved. Yet the new P-L relations are only defined up to $30^{\mathrm{d}}$, the OGLE images being saturated for LMC Cepheids with longer periods. Identification and photometry of the rare Cepheids with long periods are therefore still needed.

\subsubsection{The zero point of the $P$ - $L$ relations}

The distance modulus $(m-M)_{\mathrm{LMC}}=18.56$ (Table I), which defines the zero point of the P-L relations, has a small statistical error, but a systematic error of about \pm 0.08 cannot be excluded. The whole extragalactic distance scale would benefit from a firm LMC modulus.

\subsubsection{The metallicity effect of Cepheids}

No corrections for the dependence of Cepheid luminosities on metallicity have been applied, because even the sign of these corrections is not well known (cf. Tammann et al. 2001). Model calculations (Chiosi et al. 1993; Saio \& Gautschy 1998; Sandage et al. 1999; Alibert \& Baraffe 2000) as well as observations (Kennicutt et al. 1998; Tammann et al. 2001) indicate that the effect is smaller than $\Delta M= \pm 0.25 \Delta[\mathrm{Fe} / \mathrm{H}]$, yet a dependence of this size would still decrease/increase the known Cepheid distances by $0^{\mathrm{m}} 08(4 \%)$ on average. Independent distance information of Cepheids of different metallicity are therefore important.

\subsubsection{The width of the instability strip}

The finite width of the instability strip in the color-magnitude diagram causes an intrinsic scatter of the P-L relation which decreases with increasing wavelength (see the scatter in Fig. 1). A P-L relation gives therefore the absolute magnitude and distance of a single Cepheid to only $\sim \pm 0$. 3 . A P-L-color (P-L-C) relation of the form

$$
M=a \log P+b(B-V)+c
$$

has repeatedly been proposed to position a Cepheid within the instability strip. However, the pulsation models of Saio \& Gautschy (1998) 
have shown that the constant-period lines in the instability strip change slope in function of mass, and hence the coefficient $b$ becomes a function of period. It has also been proposed to use the amplitude to locate a Cepheid within the strip (Sandage \& Tammann 1971). Lack of sufficient data has brought these attempts to a preliminary halt.

\subsection{Difficulties with RR Lyrae Stars}

As stated before the calibration of the terms $a$ and $b$ in equation (13) is difficult, if not to say controversial. Particularly statistical parallaxes of Galactic RR Lyrae stars, which strongly depend on the sample definition, have confused the issue. But there remains a tendency of RR Lyr's - although sometimes exaggerated - to yield a smaller LMC modulus than Cepheids (cf. Table I). There is still the possibility that an additional parameter influences the RR Lyr luminosities.

\subsection{Securing the TRGB Calibration}

The luminosity calibration of the $I$-band TRGB rests so far only on a few adopted globular cluster distances, or otherwise has to rely on the Cepheid distances of external galaxies. Consequently the insensitivity of the TRGB against metallicity is not well tested yet. A definitive calibration would make the TRGB a powerful tool requiring relatively little observing time.

\subsection{Improving the SNe Ia Calibration}

The large-scale value of $H_{0}$ depends entirely on the absolute magnitudes of SNe Ia (equation 17), whose goodness in turn depends on nine calibrating Cepheid distances. Any improvement of the P-L relation of Cepheids reflects therefore directly on $H_{0}$ (cosmic). - Additional $\mathrm{SNe}$ Ia at intermediate distances are needed to tighten the correlation of their absolute magnitudes $M_{\max }$ with their decline rate $\Delta m_{15}$ and intrinsic color $(B-V)$. Allowance for this correlation only develops the full power of SNeIa as standard candles, i.e. it reduces the observed scatter of their Hubble diagram from $0 \mathrm{~m} 17$ to $0 \mathrm{~m} 11$. But the correction of $M_{\max }$ (observed) for second parameters has also a systematic effect on $H_{0}$. This is because the nine calibrating SNe Ia lie necessarily in late-type spirals (to contain Cepheids), and SNe Ia in spirals tend to be brighter and bluer and to have smaller decline rates than average (cf. Parodi et al. 2000). 


\section{GAIA's Impact on the Extragalactic Distance Scale}

\subsection{Distances of nearby Galaxies}

GAIA will measure the trigonometric parallaxes of countless stars in LMC and SMC as well as in UMi and in at least seven other nearby dwarf galaxies. This will not only provide fundamental distances of these galaxies but also fix their orientation in space.

GAIA will in addition provide rotational parallaxes for some nearby galaxies like M31 and M 33 and marginally even for galaxies outside the Local Group like NGC 55, NGC 247, NGC 253, NGC 300, M 81 and NGC 2403, by comparing their rotation curves observed from radial velocities (probably best observed from the ground) and from proper motions.

The direct distance measurements of nearby galaxies - important for themselves - will offer a control of the distance indicators discussed in the next Section.

\subsection{The Calibration of Distance Indicators}

GAIA will determine trigonometric parallaxes of thousands of Galactic Cepheids, RR Lyrae stars, and of stars at the TRGB. It will also provide highly reliable ZAMS positions in function of metallicity, and the position of the cooling sequence of White Dwarfs (WD). Moreover, GAIA will measure the parallaxes of most of the 140 Galactic Globular Clusters (GC) to better than 1\%. Their luminosity function (GCLF) may in turn become valuable for an extragalactic distance scale based on only old objects (cf. 2.2.1). Finally GAIA will decide to what extent the P-L relation of Mira variables can be made useful for distance determinations.

The above distance indicators are interlocked in a multiple way (Fig. 4). RR Lyrae and TRGB stars as well as ZAMS and WD fitting will yield distances of GCs whose trigonometric parallaxes will be known. Distances to LMC, SMC, and other nearby galaxies with known trigonometric and some rotational parallaxes will be measured again by Cepheids and RR Lyrae and TRGB stars. This network of independent distance determinations will clarify the metallicity dependence of each method. Any discrepancies which may occur will lead to new astrophysical insights.

\subsection{Intermediate Galaxy Distances from SNe Ia}

GAIA offers a unique opportunity to discover "nearby" SNeIa $(v<$ $10000 \mathrm{~km} \mathrm{~s}^{-1}$ ). Each SN Ia with good photometry yields the chance 


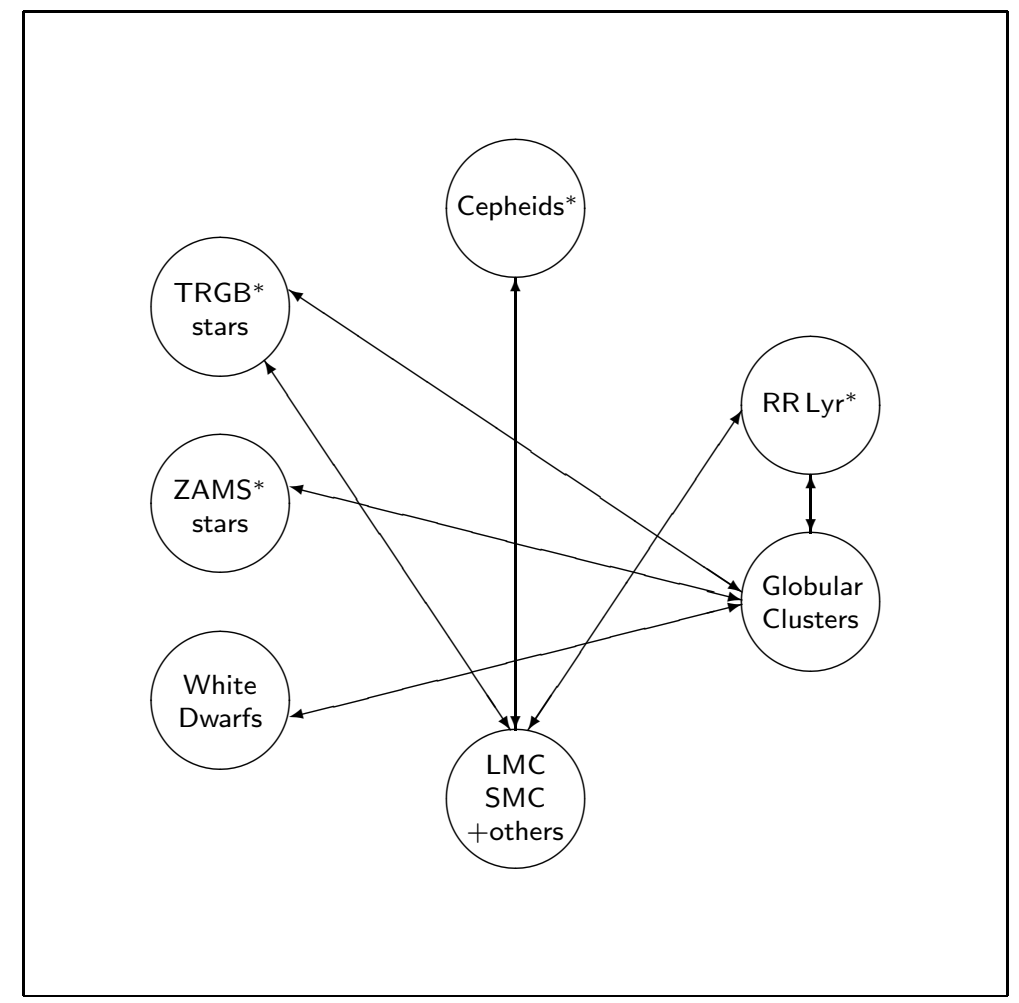

Figure 4. Objects for which GAIA will determine trigonometric parallaxes and which in turn are important for the distance scale are shown inside circles with arrows indicating their interdependencies. Stars denote distance indicators whose metallicity dependencies will be also determined by GAIA.

of the century to determine the distance of its host galaxy to within $0.11(5 \%)$. No other distance indicator can give the same accuracy, except galaxies with $v>10000 \mathrm{~km} \mathrm{~s}^{-1}$ for which the best distance is obtained from its recession velocity and by assuming a (correct) value of $H_{0}$ (cf. Section 3).

Therefore SNeIa with $v \leq 10000 \mathrm{~km} \mathrm{~s}^{-1}$ are of particular interest. Out to this limit their number can be estimated from their frequency per unit luminosity and from the luminosity density summed over all galaxies (both scaled to [for instance] $H_{0}=60$ ). The former is $\sim 0.22$ SNe Ia per $10^{10}$ solar luminosities $\left(L_{\odot}\right)$ and per 100 years, independent of the galaxy type (Tammann et al. 1994); the total galaxian luminosity density is $1.3 \cdot 10^{8} \mathrm{~L}_{\odot} \mathrm{Mpc}^{-3}$ (Tammann 1982). The volume $V\left(<10000 \mathrm{~km} \mathrm{~s}^{-1}\right)$ being $19.4 \cdot 10^{6} \mathrm{Mpc}^{3}$ yields then 555 SNe Ia per year with an error of probably less than a factor 2 . All of them have $m_{\max }^{B, V} \lesssim 16.6 \pm \sigma_{M}$, where $\sigma_{M}=0{ }^{\mathrm{m}} 2$ for SNe Ia before correction for 
decline rate and color. Half of these SNe Ia will be caught by GAIA, which covers every point in the sky once per month, near maximum, i.e. earlier than 10 days after maximum. At this time a SN Ia has faded to $m^{B, V} \lesssim 17.0$ assuming the standard light curve of Leibundgut (1991), and if it is subsequently followed for 30 days, even the faintest SN Ia of the sample will have $m^{B, V} \lesssim 19.0$. This follow-up is sufficient to extrapolate $m_{\max }^{B, V}$ and to determine the decline rate $\Delta m_{15}$ and $(B-V)_{\max }$ with sufficient accuracy even for SNe Ia whose maxima have been missed by 10 days. The resulting $m_{\max }^{B, V}$ corrected for $\Delta m_{15}$ and $(B-V)_{\max }$ will yield, if combined with $M_{\max }^{B, V}$ in equation (17), unrivalled distances for 1100 galaxies during the 4-year operation time of GAIA.

This number is overoptimistic because of Galactic absorption. Roughly one third of the SNe Ia will be at low Galactic latitudes, i.e. $|b|<20^{\circ}$ and be dimmed by $A_{V} \gtrsim 0$. 4 . They are either missed, or their photometry is impaired by large absorption corrections. SNe Ia at higher $|b|$ will suffer an absorption of $A_{V} \sim 0.25$ on average (cf. Schlegel et al. 1998). A corresponding margin of the detection and follow-up limits should therefore be allowed for. Moreover, many day time SNe Ia will not be suitable for follow-up from the ground.

Nevertheless the benefit of this programme is fourfold:

(1) The precision distances of these galaxies will map the not so local velocity field and explain the origin of the CMB dipole in great detail;

(2) The residuals of the new SNe Ia from the mean Hubble line will fix the dependencies of $m_{\max }^{B, V}$ on $\Delta m_{15}$ and $(B-V)_{\max }$ with any desiderable accuracy. These dependencies rest now only on 35 SNe Ia and their errors can still cause systematic errors of all SNe Ia distances of $3-4 \%$ (cf. Parodi et al. 2000).

(3) The new SNe Ia will richly populate the gap of the Hubble diagram (right side of Fig. 3) and fix the position of the Hubble line to within a vanishingly small error, reducing still further the random error of the constant term $c$ in equation (16) and correspondingly also of $H_{0}$. Moreover, the position of the Hubble line at $v \lesssim 10000 \mathrm{~km} \mathrm{~s}^{-1}$ is essential when the cosmological constant $\Lambda$ shall be derived from the Hubble diagram of very distant SNe Ia.

(4) The new SNe Ia, for which one will have reliable survey times, are ideally suited for the determination of their frequency with unprecedented accuracy.

GAIA cannot provide the follow-up photometry of the newly discovered SNe Ia. The above SNe Ia program is therefore only meaningful if it will be possible to organize a dedicated (earth-bound) program for the photometry in $B, V$ (and $I$ ) of the new SNe Ia down to $\sim 19$ m 0 . 
If one wants to carry the GAIA search for SNeIa to still fainter limits their total number per year increases as

$$
\log N_{\text {SNeIa }}=2.44+0.6\left(m_{\text {limit }}-17.0\right)
$$

(cf. Høg et al. 1999).

The SN sample provided by GAIA will be little "contaminated" by $\mathrm{SNe}$ II/Ib because they are fainter by $2{ }^{\mathrm{m}} 5$ on average than SNe Ia. Therefore all magnitude-limited SN searches are strongly biased against $\mathrm{SNe} I I / \mathrm{Ib}$ in spite of their considerably higher frequency per unit volume. Their value for cosmology is much smaller because their luminosity function is broad ( $\sigma_{M}=1 \mathrm{~m} 2$; Tammann \& Schröder 1990) and even skewed towards fainter SNeII (Woltjer 1998). They can hence not be used as standard candles. The determination of their individual distances from the expanding photosphere method (EPM) poses formidable problems due to the Compton scattering in moving atmospheres. Available results may therefore still be affected by systematic effects (cf. Hamuy et al. 2001 and references therein).

GAIA's high resolution power will reveal a large number of gravitationally lensed double or multiple quasars. In principle they offer themselves for an independent distance determination, but the solution is typically degenerate as to the distance and the lensing mass. Thus a single lensed quasar leaves a wide margin for $H_{0}$ (Saha \& Williams 2001). A combined solution for six quasars gives $50<H_{0}<60$ (Saha 2002). This value can be much tightened when additional cases will be discovered.

\section{Conclusions}

Reliable distance indicators (Cepheids, TF distances of complete samples, and nearby cluster distances) require a local value of $H_{0}=59.2 \pm$ 1.4. Cepheid-calibrated SNe Ia give a large-scale value of $H_{0}=57.4 \pm$ 2.3. The two values are statistically indistinguishable and one may assume $H_{0}=58 \pm 2$ everywhere.

The quoted error is only the statistical error. But the solution for $H_{0}$ is dominated by systematic errors which could amount to as much as $10 \%$. The largest systematic errors are introduced by Cepheids, which form the basis of the local as well as of the large-scale expansion rate. Equation (12) suggests, in case of a non-linear form of the P-L relation of Cepheids, that $H_{0}$ is increased by $6 \%$ for Cepheids with a median period of 30 days, i.e. a typical period for extra - Local Group galaxies. A metallicity dependence of the Cepheid P-L relation could affect $H_{0}$ by $\sim 5 \%$; if the metallicity correction of Kennicutt et al. (1998) is taken 
at face value the effect would go in the direction of decreasing $H_{0}$. The zero point of the P-L relation seems to be well determined (Table I), but a systematic error of 3-4\% cannot be excluded.

The three systematic error sources of Cepheid distances, which propagate with almost full weight into the entire extragalactic distance scale, will essentially be eliminated by GAIA.

The next important source of a systematic error - at least for the large-scale value of $H_{0}$ - comes from the homogenization of SNe Ia as to decline rate $\Delta m_{15}$ and color $(B-V)$. In fact errors of the slope of the $M-\Delta m_{15}$ and $M-(B-V)$ relations could introduce systematic errors of the SNe Ia distances of $\sim 3 \%$ (Parodi et al. 2000). This problem can entirely be solved if good photometry will be obtained for the many hundreds of SNeIa to be discovered within $10000 \mathrm{~km} \mathrm{~s}^{-1}$ by GAIA. This at the same time will yield irreplaceable distances to an equal number of field galaxies (to within random errors of $\pm 5 \%$ ), which will outline any concerted deviations from pure Hubble flow out to $10000 \mathrm{~km} \mathrm{~s}^{-1}$.

The one remaining systematic error of $\lesssim 4 \%$ comes from the difficult photometry of extra-Local Group Cepheids with the wide-field camera (WFPC-2) of HST. GAIA cannot offer a handle on this problem, and it has to await future photometry from space.

GAIA will not only much improve the value of $H_{0}$, but also the age of the oldest objects. Definitive distances to globular clusters will reduce the error of their ages, and the improvement of the distances of Local Group galaxies and the measurement of their proper motions are very important for the determination of the dynamical age of the Local Group (Lynden-Bell 1999). Thus $H_{0}$ and a minimum age of the Universe can be used as strong priors for the CMB fluctuation spectrum (cf. Netterfield et al. 2002; Pryke 2001) which will narrow down the possible range of other cosmological parameters like the baryon density $\Omega_{\mathrm{b}}$, the matter density $\Omega_{\text {Matter }}$, and the cosmological constant $\Lambda$.

\section{Acknowledgements}

The authors thank the Swiss National Science Foundation for financial support.

Note added in proof (Sep. 5, 2002). It has become clear now that the P-L relation of Galactic fundamental-mode Cepheids is distinctly different from that in LMC. The Galactic relation is quite steep, the short-period Cepheids being fainter than in LMC and the long-period ones being brighter (Tammann, Sandage, \& Reindl, in preparation). 
The non-uniqueness of the P-L relations - which is probably due, at least in part, to metallicity differences - greatly increases the need for fundamental distances of nearby galaxies from GAIA.

\section{References}

Alibert, Y., \& Baraffe, I. 2000, in: The Impact of Large-Scale Surveys on Pulsating Star Research, eds. L. Szabados and D. Kurtz, ASP Conf. Ser. 203, p. 250.

Baraffe, I., \& Alibert, Y. 2001, A\&A 371, 592.

Branch, D., Fisher, A., \& Nugent, P. 1993, AJ 106, 2383.

Carroll, S.M., Press, W.H., \& Turner, E.L. 1992, ARAछA 30, 499.

Chaboyer, B., Demarque, P., Kernan, P.J., \& Krauss, L.M. 1998, ApJ 494, 96.

Chiosi, C., Wood, P.R., \& Capitanio, N. 1993, ApJS 86, 541.

Cioni, M.-R.L., van der Marel, R.P., Loup, C. \& Habing, H.J. 2000, A $\& A$ 359, 601.

Da Costa, G.S., \& Armandroff, T.E. 1990, AJ 100, 162.

Dale, D.A., Giovanelli, R., Haynes, M.P., Camusano, L.E., \& Hardy, E. 1999, AJ 118, 1489.

Feast, M.W. 1984, MNRAS 211, 51.

Feast, M.W. 1999, PASP 111, 775.

Feast, M.W., \& Catchpole, R.M. 1997, MNRAS 286, L1.

Federspiel, M., Tammann, G.A., \& Sandage, A. 1998, ApJ 495, 115.

Federspiel, M. 1999, Ph.D. Thesis, Univ. of Basel.

Freedman, W.L., et al. 2001, ApJ 553, 47.

Giovanelli, R., et al. 1997, $A J$ 113, 22.

Girardi, L., \& Salaris, M. 2001, MNRAS 323, 109.

Groenewegen, M.A.T. \& Oudmaijer, R.D. 2000, A\&A 356, 849.

Groenewegen, M.A.T. \& Salaris, R.D. 2001, A $\& A$ 366, 752.

Hamuy, M., et al. 2001, ApJ 558, 615.

Hendry, M. 2001, in: A New Era in Cosmology, eds. T. Shanks \& N. Metcalfe ASP Conf. Ser., in press.

Høg, E., Fabricius, C., \& Makarov, V.V. 1999, Baltic Astronomy 8, 233.

Kelson, D.D., et al. 2000, ApJ 529, 768.

Kennicutt, R.C., et al. 1998, ApJ 498, 181.

Kovács, G. 2000, A\&A 363, 1.

Kraan-Korteweg, R.C. 1986, A\&AS 66, 255.

Leibundgut, B. 1991, private communication.

Lynden-Bell, D. 1999, in: The Stellar Content of Local Group Galaxies, eds. P. Whitelock \& R. Cannon, IAU Symp. 192, p. 39.

Madore, B., \& Freedman, W.L. 1991, PASP 103, 933.

Madore, B., \& Freedman, W.L. 1998, ApJ 492, 110.

Netterfield, C.B., et al. 2002, ApJ 571, 604.

Pont, F. 1999, in: Harmonizing Cosmic Distance Scales in a Post-Hipparcos Era, eds. D. Egret \& A. Heck, ASP Conf. Ser. 167, p. 113.

Panagia, N. 1999, in: New Views of the Magellanic Clouds, eds. Y.-H. Chu, N. Suntzeff, J. Hesser, \& D. Bohlender, IAU Symp. 190, p. 549.

Parodi, B.R., Saha, A., Sandage, A., \& Tammann, G.A. 2000, ApJ 540, 634.

Pryke, C. 2001, in: A New Era in Cosmology, eds. T. Shanks \& N. Metcalfe ASP Conf. Ser., in press.

Romaniello, M., Salaris, M., Cassisi, S., \& Panagia, N. 2000, ApJ 530, 738. 
Saha, A., Sandage, A., Tammann, G.A., Dolphin, A.E., Christensen, J., Panagia, N., \& Macchetto, F.D. 2001, ApJ 562, 314.

Saha, P. 2002, Talk presented at a Colloquium, Basel, 26.06.2002.

Saha, P., \& Williams, L.L.R. 2001, AJ 122, 585.

Saio, H., \& Gautschy, A. 1998, ApJ 498, 360.

Sakai, S., Zwitsky, D., \& Kennicutt, R.C. 2000, AJ 119, 1197.

Sandage, A. 1993, AJ 106, 719.

Sandage, A., Bell, R.A., \& Tripicco, M.J. 1999, ApJ 522, 250.

Sandage, A., \& Tammann, G.A. 1971, ApJ 167, 293.

Schlegel, D., Finkbeiner, D., \& Davis, M. 1998, ApJ 500, 525.

Tammann, G. A. 1982, in: Landolt-Börnstein, Astronomy \& Astrophysics, Vol. 2c, eds. W. Schaifers \& H.H. Voigt (Berlin: Springer), p. 346.

Tammann, G.A. 2001, in: The Century of Space Science, eds. J. Blecker, J. Geiß, \& M.C.E. Huber, (Dordrecht: Kluwer), in press.

Tammann, G.A., Löffler, W., \& Schröder, A. 1994, ApJS 92, 487.

Tammann, G.A., Reindl, B., Thim, F., Saha, A., \& Sandage, A. 2001, in: A New Era in Cosmology, eds. T. Shanks \& N. Metcalfe ASP Conf. Ser., in press.

Tammann, G.A., \& Sandage, A. 1999, in: Harmonizing Cosmic Distance Scales in a Post-Hipparcos Era, eds. D. Egret \& A. Heck, ASP Conf. Ser. 167, p. 204.

Tammann, G.A., \& Schröder, A. 1990, A\&A 236, 149.

Theureau, G., Rauzy, S., Bottinelli, L., \& Gouguenheim, L. 1998, A $6 A$ 340, 21.

Udalski, A., et al. 1999, AcA 49, 201.

Van Leeuwen, F., et al. 1997, MNRAS 287, 955.

Walker, A.R. 1992, ApJ 390, L81.

Walker, A. 1999, in: Post-Hipparcos cosmic candles, eds. A. Heck and F. Caputo, (Dordrecht: Kluwer), p. 125.

Woltjer, L. 1998, in: Supernovae and Cosmology, eds. L. Labhardt, B. Binggeli and R. Buser (Basel: Univ. Basel), p. 87. 